\title{
Duality and replicas for a unitary matrix model
}

\section{E. Brézin ${ }^{a}$ and S. Hikami ${ }^{b}$}

\author{
${ }^{a}$ Laboratoire de Physique Théorique, Ecole Normale Supérieure, \\ 24 rue Lhomond 75231, Paris, Cedex 05, France ${ }^{1}$ \\ ${ }^{b}$ Department of Basic Sciences, University of Tokyo, \\ Meguro-ku, Komaba, Tokyo 153, Japan \\ E-mail: brezin@lpt.ens.fr, hikami@dice.c.u-tokyo.ac.jp
}

\begin{abstract}
In a generalized Airy matrix model, a power $p$ replaces the cubic term of the Airy model introduced by Kontsevich. The parameter $p$ corresponds to Witten's spin index in the theory of intersection numbers of moduli space of curves. A continuation in $p$ down to $p=-2$ yields a well studied unitary matrix model. The application of duality and replica to the $p$-th Airy model provides, through this equivalence, a generating function for both the weak and the strong coupling expansions of the unitary model. We thereby recover and extend further the results for these expansions.
\end{abstract}

Keywords: Matrix Models, Integrable Field Theories

ARXIV EPRINT: 1005.4730

\footnotetext{
${ }^{1}$ Unité Mixte de Recherche 8549 du Centre National de la Recherche Scientifique et de l'École Normale Supérieure.
} 


\section{Contents}

1 Introduction 1

2 Dual model and its $l$-point functions $\quad 3$

3 Weak coupling expansion 5

4 Exact calculation, Virasoro constraints and comparison 6

5 Strong coupling expansion $\quad 9$

$\begin{array}{lll}6 & \text { Summary } & 13\end{array}$

\section{Introduction}

The unitary matrix model with an external source is defined by the integral over the unitary group $\mathrm{U}(N)$

$$
Z=\int d U e^{\operatorname{tr}\left(U C^{\dagger}+U^{\dagger} C\right)}
$$

where $U$ is a $N \times N$ unitary matrix, $U^{\dagger} U=1$. The external source matrix $C$ is a given fixed complex matrix. Gross and Witten [1] have studied the case in which $C$ is a multiple of the identity, $C=N / g^{2}$, and showed that, in the large $\mathrm{N}$ limit, there is a third order transition at some critical $g_{c}$. Brézin and Gross [2] generalized the model to include an arbitrary source matrix $C$ and again found that there is a phase transition governed by the parameter $\operatorname{tr}\left(C^{\dagger} C\right)^{-1 / 2}$. This model has a weak coupling expansion, when the eigenvalues $\lambda_{i}$ of $C^{\dagger} C$ are large, which involves powers and products of parameters

$$
t_{m}=\sum_{i} \frac{1}{\lambda_{i}^{m-1 / 2}}=\operatorname{Tr}\left(C^{\dagger} C\right)^{-(m-1 / 2)} .
$$

It has also a strong coupling expansion in powers of products of the parameters $\operatorname{Tr}\left(C^{\dagger} C\right)^{m}$. The strong coupling expansion is straightforward and the coefficients involving powers of the $\lambda^{m}$ have been tabulated long ago, up to $m=5$ [3]. The weak coupling expansion can be found by different methods; Gross and Newman have used Virasoro equations [4] and we reproduce their analysis in section 4 . We will follow here a completely different approach: we shall based the analysis on another matrix model, which will be shown to be equivalent to (1.1), whose interest is to show that the coefficients of the expansion of $\log Z$ in powers of the $t_{m}$ 's are related to topological invariants, namely the intersection numbers of the moduli space of $p$-spin curves on a Riemann surface, in the singular limit $p \rightarrow-2$. The same model will also be used to recover and extend the results of the strong coupling expansion. 
Indeed we shall demonstrate a remarkable equivalence of the unitary model (1.1), with a matrix model given by an integral over $N \times N$ Hermitian matrices

$$
Z_{B}=\int d B e^{\operatorname{tr} \frac{1}{B}+k \operatorname{trl} \log B+\operatorname{tr} B \Lambda}
$$

where $B$ is a $N \times N$ Hermitian matrix, $\Lambda=C C^{\dagger}$, provided one tunes properly the parameter $k$. This equivalence was already discussed by Mironov et al. [5, 6] through Virasoro equations and determinant formulations. We will show that one can recover it from an explicit determinantal expression in terms of Bessel functions [7], and from a comparison between the expansions based on (1.3) with that of (1.1). However the equivalence requires some proviso, as the reader, puzzled by the singularities due to vanishing eigenvalues of $B$, may guess. We are in fact dealing here with matrices which are unitarily equivalent to complex diagonal matrices. Therefore if we wrote $B=U X U^{\dagger}$, where $X$ is diagonal with complex entries, the integral over the unitary group would yield a Jacobian which is the modulus square of the Vandermonde determinant $J=|\Delta(X)|^{2}=\prod_{i<j}\left|x_{i}-x_{j}\right|^{2}$ and the integration over the $x_{i}$ s are over specified contours in the complex plane.

Although the equivalence holds only if the coefficient $k$ goes to zero in the weak coupling limit and to $-N$ in the strong coupling limit, we keep it arbitrary at this stage. The weak coupling expansion for $k=0$ gives the intersection numbers of the moduli space, and for $k \neq 0$, the expansion is related to the intersection numbers of the discretized moduli space [8]. The expression of (1.3) is the special case, $p=-2$ and $r=-1$, of a $(p, r)$ model

$$
Z_{(p, r)}=\int d B \exp \left(-\frac{1}{p+1} \operatorname{tr} B^{p+1}+\frac{k}{r+1} \operatorname{tr} B^{r+1}+\operatorname{tr} B \Lambda\right)
$$

which generalizes Kontsevich model [9] $p=2, k=0$. The model that we want to study involves an analytic continuation from positive integer values of $p$ and $r$ to negative values.

The reason that we find the model (1.3) interesting, in addition of the topological invariants that it allows one to compute, is that it leads to completely different ways of calculating explicit expressions for the expansions. In our previous work we have discussed a powerful duality between expectation values of characteristic polynomials [10-13], namely the relation:

$$
\int_{n \times n} d M \prod_{\alpha=1}^{N} \operatorname{det}\left(\lambda_{\alpha}-M\right) e^{-\frac{1}{2} \operatorname{tr} M^{2}+\operatorname{tr} M A}=\int_{N \times N} d B \prod_{j=1}^{n} \operatorname{det}\left(a_{j}-i B\right) e^{-\frac{1}{2} \operatorname{tr} B^{2}+\operatorname{tr} B \Lambda}
$$

where $\Lambda=\operatorname{diag}\left(\lambda_{1}, \ldots, \lambda_{N}\right)$ and $A=\operatorname{diag}\left(a_{1}, \ldots, a_{n}\right)$. Both sides are integrals over Hermitian matrices (properly normalized). The left-hand side is an $N$ point function for $n \times n$ matrices in an external given matrix source $A$, whereas the r.h.s. is an $n$-point function for $N \times N$ matrices, in the matrix source $\Lambda$. The identity (1.5) generalizes the relation between Hermitian and Kontsevich-Penner models at $A=0[14,15]$. The strategy that we use consists of the following successive steps:

- one tunes the numbers $a_{j}$ in the r.h.s. in order to generate, in an appropriate large $n$ scaling limit, the $(p, r)$ model (1.4). 
- in the Gaussian model of the left-hand side we know explicit exact expressions for the correlators $[10,16]$

$$
U\left(s_{1}, \ldots, s_{l}\right)=<\operatorname{tr} e^{s_{1} M} \operatorname{tr} e^{s_{2} M} \cdots \operatorname{tr} e^{s_{l} M}>.
$$

in which the expectation value is meant with the normalised weight proportional to $e^{-\frac{1}{2} \operatorname{tr} M^{2}+\operatorname{tr} M A}$.

- the replica method, which consists of repeating the eigenvalue $\lambda_{\alpha}, n_{\alpha}$ times and to let $n_{\alpha}$ go to zero. This is used to relate the characteristic polynomials of the l.h.s. to the usual correlation functions as in

$$
\lim _{N \rightarrow 0} \frac{1}{N} \frac{d}{d \lambda}(\operatorname{det}(\lambda-M))^{N}=\operatorname{tr} \frac{1}{\lambda-M}
$$

The resolvent is related by Fourier transform to tre $e^{s M}$ which enters into (1.6). We will explain later the meaning of taking an $N=0$ limit here in spite of the fact that in the right-hand-side of (1.5) $N$ is fixed.

In this article, we use this strategy and tune the external source $A$ to generate the $(p, r)$ model of (1.4). The limit $p=-2$ and $r=-1$, obtained through a nontrivial continuation from positive values of $p$ and $r$, is of particular interest. This rather involved construction is finally compared with the known expansions of the unitary model $Z_{U}$ of (1.1) [3-5] both in the strong and in the weak coupling phases. Our results provide series expansion for intersection numbers with $l$ marked points and arbitrary genus. The consistency with known results confirms that the duality plus replica method may be succesfully extended to negative values of $p$ and $r$ in the $(p, r)$ model (1.4).

\section{$2 \quad$ Dual model and its $l$-point functions}

The starting point is the duality relation (1.5)

$$
\int_{n \times n} d M \prod_{\alpha=1}^{N} \operatorname{det}\left(\lambda_{\alpha}-M\right) e^{-\frac{1}{2} \operatorname{tr} M^{2}+\operatorname{tr} M A}=\int_{N \times N} d B \prod_{j=1}^{n} \operatorname{det}\left(a_{j}-i B\right) e^{-\frac{1}{2} \operatorname{tr} B^{2}+\operatorname{tr} B \Lambda}
$$

where $\Lambda=\operatorname{diag}\left(\lambda_{1}, \ldots, \lambda_{N}\right)$ and $A=\operatorname{diag}\left(a_{1}, \ldots, a_{n}\right)$; the proof is simple and can be found in [10]. We now tune the $a_{j}$ in the r.h.s. to produce the model (1.4) . We use the identity

$$
\prod_{j=1}^{n} \operatorname{det}\left(1-\frac{i}{a_{j}} B\right)=\exp \left(-\sum_{j=1}^{n} \sum_{m=0}^{\infty} \frac{i^{m}}{m a_{j}^{m}} \operatorname{tr} B^{m}\right)
$$

and fix now the sums $\sum_{j=1}^{n} \frac{1}{a_{j}^{m}}$. Let us call $\rho$ the first one $(m=1)$

$$
\rho=i \sum_{j} \frac{1}{a_{j}}
$$

which may be absorbed by a shift of $\Lambda$. Taking next $\sum_{j=1}^{n} \frac{1}{a_{j}^{2}}=1$ the term $-\frac{1}{2} \operatorname{tr} B^{2}$ in the exponential is cancelled. Assume $p<r$; we can cancel all the terms other than $\operatorname{tr} B^{p}$ and 
$\operatorname{tr} B^{r}$ by chosing the $a_{j}$ so that $\sum_{j=1}^{n} \frac{1}{a_{j}^{m}}=0$, for all $m<r$, except $m=2$ and $m=p$. Note that this requires in general complex $a_{j}$. In order to eliminate the terms with $m$ larger than $r$, we have to consider the following large $n$ scaling limit:

- assume each $a_{j}$ is of order $n^{1 / 2}$ so that $\sum_{j} \frac{1}{a_{j}^{2}}=O(1)$ and in fact we chose them so that this last sum is exactly equal to one.

- we take the $\lambda_{\alpha}$ near $\rho$ in a range in which $\lambda_{\alpha}-\rho \sim n^{-1 / 2+2 / r}$

- we take the matrix $B$ of order $n^{1 / 2-2 / r}$. Then one has $\left(\sum 1 / a_{j}^{r}\right) \operatorname{tr} B^{r}$ of order one

- in this scale $\sum 1 / a_{j}^{m} \operatorname{tr} B^{m} \sim n^{2(1-m / r)}$ goes to zero for $m>r$ and one can drop all the powers higher than $q$.

- however the term $\sum 1 / a_{j}^{p} \operatorname{tr} B^{p} \sim n^{2(1-p / r)}$ grows with $n$, since $p<r$ and we have to tune the $a_{j}$ so that $\sum 1 / a_{j}^{p}$ instead of being of order $n^{1-p / 2}$ is of order $n^{1-p / 2-2(1-p / r)}$.

In this scaling limit, with the $a_{j}$ chosen as indicated, the r.h.s. gives the model $Z_{(p, r)}$ of (1.4).

The method consists of using now the left hand side of (2.1). There the duality left us with the Gaussian model

$$
Z_{\text {dual }}=\int_{n \times n} d M e^{-\frac{1}{2} \operatorname{tr} M^{2}+\operatorname{tr} M A}
$$

with the matrix $A$ fixed as just discussed above. The reason for using this dual model is that this we have for (2.4), explicit expressions for the $l$-point correlation functions

$$
U\left(s_{1}, \ldots, s_{l}\right)=<\operatorname{tr} e^{s_{1} M} \operatorname{tr} e^{s_{2} M} \cdots \operatorname{tr} e^{s_{l} M}>
$$

where the average stand for

$$
<X>=\frac{1}{Z_{\text {dual }}} \int d M X(M) e^{-\frac{1}{2} \operatorname{tr} M^{2}+\operatorname{tr} M A}
$$

The result, derived in $[10,11]$, is

$$
U\left(s_{1}, \ldots, s_{l}\right)=\int \prod_{i=1}^{l} \frac{d u_{i}}{2 i \pi} \prod_{i=1}^{l} \prod_{j=1}^{n}\left(1+\frac{s_{l}}{u_{l}-a_{j}}\right) \operatorname{det} \frac{1}{\left(u_{m_{1}}-u_{m_{2}}+s_{m_{1}}\right)} e^{\sum u_{l} s_{l}+\frac{1}{2} \sum s_{l}^{2}}
$$

in which the contour integrals circle around all the poles $a_{i}$.

Let us first consider the one point function $U(s)$.

$$
\begin{aligned}
U(s) & =\frac{e^{\frac{s^{2}}{2}}}{s} \oint \frac{d u}{2 i \pi} e^{s u} \prod_{j=1}^{n}\left(\frac{u+s-a_{j}}{u-a_{j}}\right) \\
& =\frac{1}{s} \oint \frac{d u}{2 i \pi} e^{\frac{1}{2} s^{2}+s u+\sum \log \left(\frac{u+s-a_{j}}{u-a_{j}}\right)}
\end{aligned}
$$


We use now the $a_{j}$ tuned as discussed above. Expanding the logarithm, we have

$$
\log \left(\frac{a_{j}-u-s}{a_{j}-u}\right)=-s \sum \frac{1}{a_{j}}-\frac{1}{2}\left(s^{2}+2 u s\right)-\sum_{m=3}^{\infty} \frac{1}{m} \sum_{j=1}^{n} \frac{1}{a_{j}^{m}}\left((u+s)^{m}-u^{m}\right)
$$

Using the previous tuning for the $a_{j}$ in the large- $n$ scaling limit we obtain

$$
U(s)=\frac{e^{i s \rho}}{s} \oint \frac{d u}{2 i \pi} e^{\frac{1}{p+1}\left((u+s)^{p+1}-u^{p+1}\right)+\frac{k}{r+1}\left((u+s)^{r+1}-u^{r+1}\right)}
$$

For $l$-point function $U\left(s_{1}, \ldots, s_{l}\right)$, we obtain similarly.

$$
\begin{aligned}
U\left(s_{1}, \ldots, s_{l}\right)= & \oint \prod_{j=1}^{l} \frac{d u_{j}}{2 i \pi} e^{\frac{1}{p+1} \sum_{j}\left(\left(u_{j}+s_{j}\right)^{p+1}-u_{j}^{p+1}\right)+\frac{k}{r+1} \sum_{j}\left(\left(u_{j}+s_{j}\right)^{r+1}-u_{j}^{r+1}\right)} \\
& \times \operatorname{det} \frac{1}{u_{i}-u_{k}+s_{i}} .
\end{aligned}
$$

We have studied earlier the case in which $k=0$ and $p>0$; there the $l$-point correlation functions $U\left(s_{1}, \ldots, s_{l}\right)$ are the generating function of the intersection numbers of the moduli space of curves with $p$-spins $[12,13]$.

\section{$3 \quad$ Weak coupling expansion}

We now study the model in which one continues from integer positive $p$ and $r$ down to $p=-2$ and $r=-1$. We have in this case,

$$
U(s)=\frac{1}{s} \oint \frac{d u}{2 i \pi} e^{-\frac{1}{u+s}+\frac{1}{u}+k \log (u+s)-k \log u}
$$

In the original unitary model (1.1), a simple model of one plaquette lattice QCD, the matrix $C$ is inversely proportional to the gauge coupling constant. Therefore the eigenvalues $\lambda_{\alpha}$ of $C^{\dagger} C$ are large in weak coupling, small in strong coupling. The variables in $U(s)$ is a Laplace transform of $\lambda$; therefore the weak coupling limit corresponds to large $\lambda$, hence small $s$. We first rescale $u \rightarrow s u$.

$$
U(s)=\oint \frac{d u}{2 i \pi} e^{\frac{1}{s u(u+1)}}\left(\frac{u+1}{u}\right)^{k},
$$

since we continue later in $k$ down to negative values, the contour integral is really an integral over the discontinuity along the real $u$-axis. We chose a countour in the $u$-plane which goes parallel to the imaginary axis through the point $u=-1 / 2$ at which $1 / u(u+1)$ is maximum in the real direction and change variable $u=1 / 2(-1+i / x \sqrt{s})$ :

$$
U(s)=-\frac{1}{4 \pi \sqrt{s}} \int \frac{d x}{x^{2}} e^{-\frac{4 x^{2}}{1+s x^{2}}}\left(\frac{1-i x \sqrt{s}}{1+i x \sqrt{s}}\right)^{k}
$$

This integral contains a singular term proportional to $1 / \sqrt{s}$ which we discard, i.e. in order to obtain the terms of the expansion with positive powers of $s$, we consider instead

$$
U_{+}(s)=-\frac{1}{4 \pi \sqrt{s}} \int \frac{d x}{x^{2}}\left[e^{-\frac{4 x^{2}}{1+s x^{2}}}\left(\frac{1-i x \sqrt{s}}{1+i x \sqrt{x}}\right)^{k}-e^{-4 x^{2}}\right]
$$


For $s$ small, we can expand the integrand in powers of $s$ and compute the successive coefficients as Gaussian integrals. The result for the positive powers of $s$ takes the form

$$
\begin{aligned}
U_{+}(s)= & -\frac{1}{2 \sqrt{\pi s}}\left(-s \frac{1}{8}\left(4 k^{2}-1\right)+s^{2} \frac{1}{3 ! 2^{7}}\left(4 k^{2}-1\right)\left(4 k^{2}-9\right)\right. \\
& -s^{3} \frac{1}{5 ! 2^{9}}\left(4 k^{2}-1\right)\left(4 k^{2}-9\right)\left(4 k^{2}-25\right) \\
& +s^{4} \frac{1}{21 \cdot 2^{18}}\left(4 k^{2}-1\right)\left(4 k^{2}-9\right)\left(4 k^{2}-25\right)\left(4 k^{2}-49\right) \\
& \left.-s^{5} \frac{1}{135 \cdot 2^{22}}\left(4 k^{2}-1\right)\left(4 k^{2}-9\right)\left(4 k^{2}-25\right)\left(4 k^{2}-49\right)\left(4 k^{2}-81\right)+O\left(s^{6}\right)\right)
\end{aligned}
$$

If we set $k=0$ in this result the $(p, r)$ model of (1.4) reduces to the $p$-th Airy model, with here $p=-2$. Let us verify that the coefficients of $s^{m}$ in the above series (3.5) do coincide with the intersection numbers for $p=-2$ of the $p$-th generalized Kontsevich model [13]. Indeed for this model we had found

$$
\begin{aligned}
U(s)= & \frac{1}{N s^{1+\frac{1}{p}} \pi}\left[\Gamma\left(1+\frac{1}{p}\right)-\frac{p-1}{24} y \Gamma\left(1-\frac{1}{p}\right)\right. \\
& \left.+\frac{(p-1)(p-3)(1+2 p)}{5 ! 4^{2} \cdot 3} y^{2} \Gamma\left(1-\frac{3}{p}\right)+\cdots\right]
\end{aligned}
$$

where $y=s^{2+\frac{2}{p}}$. For $p=-2$, this does agree with the series (3.5) for $k=0$.

In the next section we shall compare these results with the weak coupling expansion derived both for the model defined by (1.3) and for the unitary model (1.1) and see that they agree with (3.5) in the limit $k=0$. The alleged correspondence of these two models is in fact true in the limit $k \rightarrow-N$, but in this calculation we are focusing on the terms of the weak coupling expansion which contain only one single trace. The flow of internal index has only one cycle and in the zero replica limit only those terms survive.

\section{Exact calculation, Virasoro constraints and comparison}

Instead of using duality and replica we return to the partition function $Z_{B}$ defined in (1.3). Standard techniques allow us to express $Z_{B}$ as a determinant of modified Bessel functions for arbitrary $k$. We shall compare the weak coupling expansion deduced from this explicit expression, with that obtained above from $U(s)$ using the dual model. Denoting the eigenvalues of $B$ by $x_{j}(j=1, \ldots N)$, we can integrate over the unitary group in the matrix integral (1.3). Given the non commutation of the matrices $B$ and $\Lambda$ this requires the use of the HarishChandra (Itzykson-Zuber) formula: there is thus a division of the Jacobian measure $|\Delta(x)|^{2}$ by $\Delta(x)$. Once the integration over the unitary group is done we have

$$
Z_{B}=\int \prod_{i=1}^{N} d x_{i} \frac{\overline{\Delta(x)}}{\Delta(\lambda)} \prod_{i=1}^{N} x_{i}^{k} e^{\sum_{i} \frac{1}{x_{i}}+\sum x_{i} \lambda_{i}} .
$$


in which the complex eigenvalues $x_{i}$ circle around the origin in the complex plane. Changing variables $x_{i} \rightarrow \frac{1}{\sqrt{\lambda_{i}}} e^{i \theta_{i}}$, the integrals over these angles produce modified Bessel functions $I_{m}(z)$, and $Z_{B}$ has an explicit expression as a determinant,

$$
Z_{B}=\frac{1}{\left(\prod \lambda_{i}\right)^{\frac{k+1}{2}} \Delta(\lambda)} \operatorname{det}\left(\frac{1}{\lambda_{i}^{\frac{j-1}{2}}} I_{k+j}\left(2 \sqrt{\lambda_{i}}\right)\right)_{i, j}
$$

In the weak coupling region of large $\lambda_{j}$, we use the asymptotic expansion of the modified Bessel functions:

$$
\begin{aligned}
I_{l}(2 \sqrt{\lambda})= & \frac{e^{2 \sqrt{\lambda}}}{\sqrt{4 \pi \sqrt{\lambda}}}\left(1-\frac{l^{2}-\frac{1}{4}}{4 \sqrt{\lambda}}+\frac{\left(l^{2}-\frac{1}{4}\right)\left(l^{2}-\frac{9}{4}\right)}{2 !(4 \sqrt{\lambda})^{2}}\right. \\
& \left.-\frac{\left(l^{2}-\frac{1}{4}\right)\left(l^{2}-\frac{9}{4}\right)\left(l^{2}-\frac{25}{4}\right)}{3 !(4 \sqrt{\lambda})^{3}}+\cdots\right) .
\end{aligned}
$$

Retaining only the leading terms in (4.3) for large $\lambda_{j}$, gives the term of order zero

$$
Z_{0}=\prod_{i<j}^{N} \frac{1}{\sqrt{\lambda_{i}}+\sqrt{\lambda_{j}}} \prod_{i=1}^{N} \frac{1}{\lambda_{i}^{\frac{k}{2}}} e^{\sum_{i=1}^{N} 2 \sqrt{\lambda_{i}}}
$$

This is the nothing but the genus zero contribution to the free energy $F$ found in [2] for the unitary model.

Keeping $\mathrm{N}$ finite, we can calculate a few terms in the expansion in inverse powers of $\lambda$ :

$$
\begin{aligned}
Z_{B}= & Z_{0}\left[1-\frac{(2 k+2 N)^{2}-1}{16} \sum_{i=1}^{N} \frac{1}{\sqrt{\lambda_{i}}}\right. \\
& +\frac{\left((2 k+2 N)^{2}-1\right)\left((2 k+2 N)^{2}-9\right)}{512}\left(\sum_{i=1}^{N} \frac{1}{\sqrt{\lambda_{i}}}\right)^{2} \\
& +\frac{\left((2 k+2 N)^{2}-1\right)\left((2 k+2 N)^{2}-9\right)}{3 ! 4^{6}} \\
& \left.\times\left(-8 \sum_{i=1}^{N} \frac{1}{\left(\lambda_{i}\right)^{\frac{3}{2}}}+\left((2 k+2 N)^{2}-17\right)\left(\sum_{i=1}^{N} \frac{1}{\sqrt{\lambda_{i}}}\right)^{3}\right)+\cdots\right]
\end{aligned}
$$

Note that $N$ appears always in the combination $(k+N)$. Therefore, setting $k=-N$, all the factors $(2 k+2 N)$ vanish and we find that the weak coupling expansion of the partition function $Z_{B}$ (1.3), up to this order, agrees with the weak coupling expansion obtained above in (3.5) with the dual model at $k=0$, if we look only at the single trace terms. Furthermore it agrees with the known weak coupling expansion of the unitary model [7] $Z_{U}$ defined by (1.1). This is as a manifestation of the equivalence of $Z_{U}$ in (1.1) and $Z_{B}$ in (1.3) at $k=-N$.

This same equivalence at $k=-N$ in (4.5) can be established through the weak coupling expansion based on Virasoro equations. The Virasoro equations for the partition function 
$Z_{U}$ in (1.1) have been derived by Gross and Newman [4]. We quote here their results; the unitary model satisfies the obvious equation

$$
\frac{\partial^{2} Z_{U}}{\partial C_{a b} \partial C_{b c}^{\dagger}}=\delta_{a c} Z_{U}
$$

but $Z_{U}$ is only function of the eigenvalues $\lambda_{i}$ of $C C^{\dagger}$; one thus obtains [2]

$$
\frac{\partial^{2} Z_{U}}{\partial \lambda_{a}^{2}}+\sum_{a \neq b} \frac{1}{\lambda_{a}-\lambda_{b}}\left(\frac{\partial Z_{U}}{\partial \lambda_{a}}-\frac{\partial Z_{U}}{\partial \lambda_{b}}\right)=\frac{1}{\lambda_{a}}\left(Z_{U}-\sum_{b} \frac{\partial Z_{U}}{\partial \lambda_{b}}\right)
$$

With $Z_{U}=Z_{0} Y$, the Virasoro constraints [4] take the form

$$
L_{n} Y=-\partial_{n} Y \quad n \geq 0
$$

with the $L_{n}$ given by

$$
\begin{array}{ll}
n=0: & \quad \sum_{k=0}^{\infty}\left(k+\frac{1}{2}\right) \tilde{t}_{k} \partial_{k} Y+\frac{1}{16}=-\partial_{0} Y, \\
n \geq 1: & \sum_{k=0}^{\infty}\left(k+\frac{1}{2}\right) \tilde{t}_{k} \partial_{k+n} Y+\frac{1}{4} \sum_{k=1}^{n} \partial_{k-1} \partial_{n-k} Y=-\partial_{n} Y .
\end{array}
$$

where

$$
\tilde{t}_{k}=-\frac{1}{2 k+1} \sum_{b} \frac{1}{\lambda_{b}^{k+\frac{1}{2}}}
$$

Following our previous notation for the $p$-spin curves, we use instead the parameters

$$
t_{m}=\sum_{i} \frac{1}{\lambda_{i}^{m-\frac{1}{2}}} .
$$

Thus $\tilde{t}_{0}$ corresponds to $-t_{1}$ in our notation. There is no $t_{0}$ in the expansion of $Z$ for the unitary matrix model. Using the Virasoro constraints, we can sove for the case in which all the $t_{n}$, except $t_{1}$, vanish. The equation (4.9) allows one to determine this expansion:

$$
Y=1+\frac{1}{16} t_{1}+\frac{9}{512} t_{1}^{2}+\frac{9 \times 17}{3 ! 4^{6}} t_{1}^{3}+\cdots
$$

These terms correspond to the intersection numbers for one, two and three marked points. If we set $k=-N$ in our previous result (4.5), in terms of Bessel functions, we find that the expression coincides indeed with $Y$ given by (4.12). This shows within this expansion the coincidence of the two models. Note that the successive terms are independent of $N$.The weak coupling expansion for general $k$ is given by (4.5). The equation (3.5) was missing this $k+N$, replaced by $k$; this is because we were using the replica limit to focus on single trace terms. But the true correspondence between the unitary and B-model would yield $k+N$ instead of $k$ in (3.5), as one sees on (4.5) and we have to set $k=-N$ at the end to recover the unitary model. 


\section{$5 \quad$ Strong coupling expansion}

We now turn to the strong coupling expansion ( small $\lambda_{j}$ ) for the B-model. We can first use the representation in terms of Bessel functions. Next we shall compare with the calculation based on the $l$-point correlation function $U\left(s_{1}, \ldots, s_{l}\right)$ for large $s$ and verify that they agree as expected. The ascending series for the asymptotic expansion of the modified Bessel function is given by

$$
I_{\nu}(z)=\left(\frac{1}{2} z\right)^{\nu} \sum_{m=0}^{\infty} \frac{\left(\frac{1}{4} z^{2}\right)^{m}}{m ! \Gamma(\nu+m+1)}
$$

Putting this expansion into the determinant of (4.2), we find for $k=-N$ for the unitary matrix model,

$$
\begin{aligned}
Z= & C\left(1+\frac{1}{N} \sum_{i} \lambda_{i}+\frac{1}{2\left(N^{2}-1\right)}\left(\sum_{i} \lambda_{i}\right)^{2}-\frac{1}{2 N\left(N^{2}-1\right)} \sum_{i} \lambda_{i}^{2}\right. \\
& +\frac{N^{2}-2}{6 N\left(N^{2}-1\right)\left(N^{2}-4\right)}\left(\sum_{i} \lambda_{i}\right)^{3}-\frac{1}{2\left(N^{2}-1\right)\left(N^{2}-4\right)}\left(\sum_{i} \lambda_{i}^{2}\right)\left(\sum_{i} \lambda_{i}\right) \\
& \left.+\frac{2}{3 N\left(N^{2}-1\right)\left(N^{2}-4\right)} \sum_{i} \lambda_{i}^{3}+O\left(\lambda^{4}\right)\right)
\end{aligned}
$$

where $C$ is a constant term.

We now consider the strong coupling expansion of the B-model (1.3) from the correlation functions $U\left(s_{1}, \ldots, s_{l}\right)$ in its dual representation. Strong coupling means small $\lambda$ 's. We are thus looking at the large $s$ expansion for the Fourier conjugate variable $s \sim \frac{1}{\lambda}$.

Let us begin with the one point function $U(s)$, given by (3.2). The shift $u \rightarrow(u-1) / 2$ gives

$$
U(s)=\frac{1}{2} \oint \frac{d u}{2 i \pi} e^{\frac{4}{s\left(u^{2}-1\right)}}\left(\frac{u+1}{u-1}\right)^{k} .
$$

As before there is an $s$-independent constant if we replace the exponential by one which does not affect the coefficients of the expansion, and we consider thus

$$
U_{+}(s)=\frac{1}{2} \sum_{1}^{\infty} \frac{4^{m}}{m ! s^{m}} \oint \frac{d u}{2 i \pi} \frac{1}{\left(u^{2}-1\right)^{m}}\left(\frac{u+1}{u-1}\right)^{k} .
$$

The contour integral is around the origin, but since the integrand falls off at infinity, we can replace the contour by an integral over the discontinuities accross the cuts which run from $(1, \infty)$ and $(-\infty,-1)$. Then one finds

$$
\oint \frac{d u}{2 i \pi} \frac{1}{\left(u^{2}-1\right)^{m}}\left(\frac{u+1}{u-1}\right)^{k}=-\frac{2}{\pi} \sin \pi k \int_{1}^{\infty} d x \frac{(x+1)^{k-m}}{(x-1)^{k+m}}
$$

which vanishes as it should when $k$ is a positive or negative integer. The last integral is an Euler beta function:

$$
\int_{1}^{\infty} d x \frac{(x+1)^{k-m}}{(x-1)^{k+m}}=2^{1-2 m}(2 m-2) ! \frac{\Gamma(-k-m+1)}{\Gamma(-k+m)} .
$$


We now go to $k=-N$ as discussed earlier, dropping obviously the vanishing factor $\sin \pi k$. Note that the Gamma functions give

$$
\frac{\Gamma(N-m+1)}{\Gamma(N+m)}=\prod_{l=1}^{m-1} \frac{1}{N^{2}-l^{2}}
$$

which yields only even powers of $1 / N$ in a $1 / N$-expansion, as it should for the initial unitary model. Finally, up to an additional constant and to an overall factor we find

$$
U(s)=\sum_{1}^{\infty} \frac{(2 m-2) !}{m ! s^{m}} \frac{1}{\prod_{l=1}^{m-1}\left(N^{2}-l^{2}\right)}
$$

This expression agrees, as claimed earlier, with the single trace terms of the strong coupling expansion of $\log Z$ (5.2) obtained from the determinant of Bessel functions.

The result is an asymtotic expansion, as exhibited by the presence of De Wit-'t Hooft poles in $N[17]$ for any integer $N$, which imply that the $1 / N$ expansion is certainly only asymptotic. Since the parameter $s$ is a conjugate Fourier variable, the use of one single $s$ in $U(s)$, rather than several as in $U\left(s_{1} \cdots s_{l}\right)$ limits ourselves in the strong coupling (small $\lambda)$ region to terms of the form

$$
\frac{1}{s^{m}}=\sum_{i} \lambda_{i}^{m}
$$

If we wanted to compare our results with the full result for the unitary integral we would need multiple $s_{i}$ to generate terms of the form $\sum_{i} \lambda_{i}^{m_{1}} \sum_{i} \lambda_{i}^{m_{2}} \ldots$. Therefore the expression (4.10) has to be compared with the strong coupling expansion of the unitary model in which we would keep only the terms with one single trace $\left(\sum_{i} \lambda_{i}^{m}=\operatorname{tr}\left(C^{\dagger} C\right)^{m}\right)$.

The strong coupling expansion of the unitary model has been studied long ago and we have tables for the expansion due to the work of Samuel [3] who studied the model

$$
Z_{U}^{s}=\int d U \exp \left[\beta \operatorname{tr} A U+\beta \operatorname{tr} B U^{\dagger}\right]
$$

where the $\beta$ is the coupling constant. The expansion has the form

$$
\begin{aligned}
\log Z_{U}^{s}= & N^{2} \sum_{n=1}^{\infty} \frac{(\beta)^{2 n}}{n !} \sum_{\alpha_{1}, \ldots, \alpha_{n}} N^{2 n-2} C_{\left(\alpha_{1}, \ldots, \alpha_{n}\right)}^{c}(N) \\
& \times\left(\frac{\operatorname{tr} A B}{N}\right)^{\alpha_{1}}\left(\frac{\operatorname{tr} A B A B}{N}\right)^{\alpha_{2}} \cdots\left(\frac{\operatorname{tr}(A B)^{n}}{N}\right)^{\alpha_{n}}
\end{aligned}
$$

with the restriction $\alpha_{1}+2 \alpha_{2}+\cdots+n \alpha_{n}=n$. (The superscript $c$ in $C_{\left(\alpha_{1}, \cdots \alpha_{n}\right)}^{c}$ refers to the connected part, the expansion of $\log Z_{U}$ rather than $Z_{U}$ ). The calculations of [3] have been performed up to order $\beta^{10}$. Without in fact losing generality we can substitute $\beta A=C, \beta B=C^{\dagger}$ and use those results for the unitary model (1.1). Let us first focus on the coefficients with one single trace, namely $\alpha_{n}=1$ and $\alpha_{l}=0$ for $l<n$. We denote the 
coefficient of $\operatorname{Tr}(A B)^{n}$ simply by $C_{n}$ and we find in reference [3]

$$
\begin{aligned}
& C_{1}=1, \quad C_{2}=-\frac{1}{N^{2}-1}, \quad C_{3}=\frac{4}{\left(N^{2}-1\right)\left(N^{2}-4\right)} \\
& C_{4}=-\frac{30}{\left(N^{2}-1\right)\left(N^{2}-4\right)\left(N^{2}-9\right)} \\
& C_{5}=\frac{336}{\left(N^{2}-1\right)\left(N^{2}-4\right)\left(N^{2}-9\right)\left(N^{2}-16\right)}
\end{aligned}
$$

These numbers agree completely with the coefficients of $s^{-m}$ in (5.8) and the comparison shows that at all orders the result for (5.11) would give for the single trace terms.

$$
C_{m}=(-1)^{m-1} \frac{(2 m-1) !}{m !} \frac{1}{\prod_{l=1}^{m-1}\left(N^{2}-l^{2}\right)} .
$$

If we want to use the same strategy to compare our results with the terms of the strong coupling expansion which involve product of several traces (or powers of one trace), we have to consider higher point functions. For instance the product of two traces requires now to consider the large $\left(s_{1}, s_{2}\right)$ expansion of the two point function $U\left(s_{1}, s_{2}\right)$. We know this function explicitely: it is given by the double integral (2.9). It has a connected part $U\left(s_{1}, s_{2}\right)$ which is obtained by taking the cycle of length two in the determinant:

$$
\begin{aligned}
U\left(s_{1}, s_{2}\right)= & \oint d u_{1} d u_{2} \frac{1}{\left(u_{2}-u_{1}+s_{2}\right)\left(u_{2}-u_{1}-s_{1}\right)} e^{\frac{s_{1}}{u_{1}\left(u_{1}+s_{1}\right)}+\frac{s_{2}}{u_{2}\left(u_{2}+s_{2}\right)}} \\
& \times\left(\frac{u_{2}+s_{2}}{u_{2}}\right)^{k}\left(\frac{u_{1}+s_{1}}{u_{1}}\right)^{k}
\end{aligned}
$$

We follow the same strategy: change variables $u_{1} \rightarrow s_{1} u_{1}, u_{2} \rightarrow s_{2} u_{2}$ expand the exponentials $e^{\frac{1}{s u(u+1)}}$ in powers of $1 / s$, but we have also to deal with the pole terms $\frac{1}{s_{2}\left(1+u_{2}\right)-s_{1} u_{1}}$ and $\frac{1}{s_{2} u_{2}-s_{1}\left(1+u_{1}\right)}$. There we take a dissymetric limit in which $s_{2} \gg s_{1}$ and replace

$$
\begin{aligned}
& \frac{1}{s_{2}\left(1+u_{2}\right)-s_{1} u_{1}}=\frac{1}{s_{2}} \sum_{m=0}^{\infty} \frac{u_{1}^{m}}{\left(1+u_{2}\right)^{m+1}}\left(\frac{s_{1}}{s_{2}}\right)^{m} \\
& \frac{1}{s_{2} u_{2}-s_{1}\left(1+u_{1}\right)}=\frac{1}{s_{2}} \sum_{m=0}^{\infty} \frac{\left(1+u_{1}\right)^{m}}{u_{2}^{m+1}}\left(\frac{s_{1}}{s_{2}}\right)^{m}
\end{aligned}
$$

The result is a sum of factorized contour integrals in $u_{1}$ and $u_{2}$ similar to the one that we encountered in (5.4). These integrals may be deformed into integrals of discontinuities over the real axis, and after division by the factors $\sin \pi k$ we can continue to $k=-N$. The integrals of the discontinuities are of the type

$$
\int_{0}^{\infty} d x \frac{x^{N-b}}{(1+x)^{N-a}}=B(N-b-1, b-a-1),
$$

with the Euler beta function $B(p, q)=\Gamma(p) \Gamma(q) / \Gamma(p+q)$. The coefficient of $1 / s_{1}^{m_{1}} 1 / s_{2}^{m-2}$ is then given as an explicit sum:

$$
\sum_{p_{1}, p_{2}} \frac{B\left(N-m_{1}-p_{2}, p_{1}+p_{2}+2 m_{1}+1\right) B\left(N-m_{2}+p_{1}+1,2 m_{2}-p_{1}-p_{2}-1\right)}{\left(m_{1}+p_{1}+p_{2}+1\right) !\left(m_{2}-p_{1}-p_{2}-1\right) !} .
$$


In the reference [3] we read the first coefficients $C_{1, m}$ of $\left(\frac{\operatorname{tr} A B}{N}\right)\left(\frac{\operatorname{tr}(A B)^{m}}{N}\right)$ namely

$$
\begin{aligned}
& C_{1,1}=1 /\left(N^{2}-1\right) \\
& C_{1,2}=-12 /\left(N^{2}-1\right)\left(N^{2}-4\right) \\
& C_{1,3}=120 /\left(N^{2}-1\right)\left(N^{2}-4\right)\left(N^{2}-9\right) \\
& C_{1,4}=-1680 /\left(N^{2}-1\right)\left(N^{2}-4\right)\left(N^{2}-9\right)\left(N^{2}-16\right) .
\end{aligned}
$$

We now compare with the coefficient of the term $\frac{1}{s_{2} s_{1}^{m-1}}$ in the strong coupling expansion of $U\left(s_{1}, s_{2}\right)$ (5.18). For instance the coefficient of $1 / s_{1} s_{2}^{2}$ appears as a sum of three terms

$$
\begin{aligned}
\frac{N^{2}}{s_{2}^{2} s_{1}}[ & \frac{1}{N^{2}\left(N^{2}-1\right)(N+1)(N+2)}+\frac{1}{N^{2}\left(N^{2}-1\right)(N-1)(N-2)} \\
& \left.+\frac{2}{N^{2}\left(N^{2}-1\right)^{2}}\right]=\frac{1}{s_{2}^{2} s_{1}} \frac{4}{\left(N^{2}-1\right)\left(N^{2}-4\right)} ;
\end{aligned}
$$

note that the sum if a function of $N^{2}$ as it should since the unitary integral (1.1) has an expansion in even powers of $1 / N$. Let us go further and quote from [3] the first two $C_{2, m}$ coefficients of $\left(\frac{\operatorname{tr}(A B)^{2}}{N}\right)\left(\frac{\operatorname{tr}(A B)^{m}}{N}\right)$

$$
\begin{aligned}
C_{2,2} & =\frac{18\left(3 N^{2}-7\right)}{\left(N^{2}-1\right)^{2}\left(N^{2}-4\right)\left(N^{2}-9\right)} \\
C_{2,3} & =-480 \frac{\left(3 N^{2}-13\right)}{\left(N^{2}-1\right)^{2}\left(N^{2}-4\right)\left(N^{2}-9\right)\left(N^{2}-16\right)} .
\end{aligned}
$$

The sums (5.18) lead to a coefficient of $1 / s_{1}^{2} s_{2}^{2}$ equal to

$$
\begin{gathered}
\frac{N^{2}}{s_{1}^{2} s_{2}^{2}}\left(\frac{5}{N^{2}\left(N^{2}-1\right)\left(N^{2}-4\right)(N+1)(N+3)}\right. \\
\left.\quad+\frac{5}{N^{2}\left(N^{2}-1\right)\left(N^{2}-4\right)(N-1)(N-3)}+\frac{8}{N^{2}\left(N^{2}-1\right)^{2}\left(N^{2}-4\right)}\right) \\
\quad=\frac{1}{s_{1}^{2} s_{2}^{2}} \frac{6\left(3 N^{2}-7\right)}{\left(N^{2}-1\right)^{2}\left(N^{2}-4\right)\left(N^{2}-9\right)}
\end{gathered}
$$

and for $1 / s_{1}^{3} s_{2}^{2}$

$$
\begin{aligned}
\frac{N^{2}}{s_{2}^{2} s_{1}^{3}}( & \frac{42}{N^{2}\left(N^{2}-9\right)\left(N^{2}-4\right)\left(N^{2}-1\right)(N+1)(N+4)} \\
& +\frac{42}{N^{2}\left(N^{2}-9\right)\left(N^{2}-4\right)\left(N^{2}-1\right)(N-1)(N-4)} \\
& \left.+\frac{60}{N^{2}\left(N^{2}-1\right)^{2}\left(N^{2}-4\right)\left(N^{2}-9\right)}\right) \\
& =\frac{1}{s_{2}^{2} s_{1}^{3}} \frac{48\left(3 N^{2}-13\right)}{\left(N^{2}-1\right)^{2}\left(N^{2}-4\right)\left(N^{2}-9\right)\left(N^{2}-16\right)}
\end{aligned}
$$

Again the various sterms of the sums combine and give, as expected, functions of $N^{2}$. The comparison with Samuel's results (5.21) is remarkable. Still there are combinatorial factors 
which make the agreement incomplete: for instance a factor 10 is missing in the expansion of $U\left(s_{1}, s_{2}\right)$ for $C_{2,3}$ which corresponds to the choice 5!/2!3! of assigning $s_{1}$ and $s_{2}$ to a particular trace, i.e. the number of ways of splitting 5 objects into two groups of of 2 and 3 ; a factor 3 has to multiply our coefficient of $1 / s_{1}^{2} s_{2}^{2}$, i.e. the number of ways of splitting the 4 powers in 2 and 2. More generally a factor $\left(m_{1}+m_{2}\right) ! / m_{1} ! m_{2} !$ if $m_{1} \neq m_{2}$ and $\left(2 m_{1}\right) ! / 2\left(m_{1} !\right)^{2}$ if $m_{1}=m_{2}$.

Clearly this strategy may be extended to $l$-point functions $(\geq 3)$, for any $l$. For instance, if $l=3$, we take the connected part of the 3 by 3 determinant in (2.9),

$$
\begin{aligned}
\frac{1}{\left(u_{2}-u_{1}+s_{2}\right)\left(u_{3}-u_{2}+s_{3}\right)\left(u_{1}-u_{3}+s_{1}\right)} \\
\quad+\frac{1}{\left(u_{3}-u_{1}+s_{3}\right)\left(u_{2}-u_{3}+s_{2}\right)\left(u_{1}-u_{2}+s_{1}\right)}
\end{aligned}
$$

and repeat exactly the same expansions with some ordering of $s_{1}, s_{2}, s_{3}$ as above, to obtain finally the coefficient of $s_{1}^{-m_{1}} s_{2}^{-m_{2}} s_{3}^{-m_{3}}$. For instance, we obtain at the lowest order the coefficient of $s_{1}^{-1} s_{2}^{-1} s_{3}^{-1}$ as

$$
-\frac{1}{s_{1} s_{2} s_{3}} \frac{8}{\left(N^{2}-1\right)\left(N^{2}-4\right)}
$$

which, up to a sign, coincides with $C_{1,1,1}$, the coefficient of $(\operatorname{Tr}(A B))^{3}$.

We have thus verified that the functions $U\left(s_{1}, \ldots, s_{l}\right)$ provide the generating function for the coefficients $C_{\alpha}^{c}(N)$ for finite $N$. The strong coupling expansion, in the presence of the external source $C^{\dagger} C$ has also been analysed by Virasoro type recursion relations [5]. However our explicit formulae for $U\left(s_{1}, \ldots, s_{l}\right)$, from which one may obtain both the strong coupling and the weak coupling expansions, are very powerful.

\section{Summary}

We have found that the unitary matrix model may be replaced by a generalized $p$-spin higher Airy model which yields the same weak and strong coupling expansions. After a duality which replaces this alternative $(p, r)$ model by a Gaussian model in an external matrix source, we can compute explicitely the Fourier transform of the $l$-point correlation functions $U\left(s_{1}, \ldots, s_{l}\right)(2.9)$. This provides when $p=-2$ and $r=-1$ a generating function for the weak and the strong coupling expansions of the unitary matrix model $Z_{U}$ in (1.1). The number $l$ correponds to the total number of traces involving the external source matrices $C C^{\dagger}$ in the expansions.

In the weak coupling region, the replica method provides the terms of the expansion ordered by the number of independent traces involved in the expansion. The zero-replica limit for the one-point function $U(s)$, determines the single trace terms and we have verified the agreement with the weak coupling expansion of the unitary matrix model.

In the strong coupling region, the $l$-point functions $U\left(s_{1}, \ldots, s_{l}\right)$ remarkably produce the correct results of the strong coupling expansion of the unitary matrix model with an external source, with its involved $N$-dependence, De Wit-'t Hooft poles, and allows one to go much beyond the terms which had been tabulated long ago. 
Therefore this other matrix model provides finite integrals, on one variable for the single trace terms, two variables for product of two traces, etc., which yield both the weak and the strong coupling regions. It also shows an unexpected connexion between the unitary matrix model and the generalized Kontsevich model which we know is a generating function for intersection numbers [9, 18-20].

The Gross-Witten phase transition between the two regions may also be recovered on the integrals for $U(s)$; it comes from the logarithmic potential of the matrix model (1.3). The applications of these techniques to other values of $p$ than -2 with a logarithmic potential may be interesting, and it leads also to integral representations for $U\left(s_{1}, \ldots, s_{l}\right)$ as in (2.9).

Finaly let us note the phase transition in the unitary matrix model has been discussed over the last years by several authors in the context of string/black hole physics [21-23]. The reformulation presented here may be of interest in this other context as well.

\section{Acknowledgments}

S.H. is supported by Grant-in Aid for Scientific Research(C) of JSPS.

Open Access. This article is distributed under the terms of the Creative Commons Attribution Noncommercial License which permits any noncommercial use, distribution, and reproduction in any medium, provided the original author(s) and source are credited.

\section{References}

[1] D.J. Gross and E. Witten, Possible third order phase transition in the large- $N$ lattice gauge theory, Phys. Rev. D 21 (1980) 446 [SPIRES].

[2] E. Brézin and D.J. Gross, The external field problem in the large- $N$ limit of $Q C D$, Phys. Lett. B 97 (1980) 120 [SPIRES].

[3] S. Samuel, $\mathrm{U}(N)$ Integrals, $1 / N$, and the De Wit-'t Hooft anomalies,

J. Math. Phys. 21 (1980) 2695 [SPIRES].

[4] D.J. Gross and M.J. Newman, Unitary and Hermitian matrices in an external field. 2: The Kontsevich model and continuum Virasoro constraints, Nucl. Phys. B 380 (1992) 168 [hep-th/9112069] [SPIRES].

[5] A. Mironov, A. Morozov and G.W. Semenoff, Unitary matrix integrals in the framework of generalized Kontsevich model. 1: Brezin-Gross-Witten model, Int. J. Mod. Phys. A 11 (1996) 5031 [hep-th/9404005] [SPIRES].

[6] A. Alexandrov, A. Mironov and A. Morozov, BGWM as second constituent of complex matrix model, JHEP 12 (2009) 053 [arXiv: 0906.3305] [SPIRES].

[7] R. Brower, P. Rossi and C.-I. Tan, The external field problem for QCD, Nucl. Phys. B 190 (1981) 699 [SPIRES].

[8] L. Chekov, Matrix model for discretized moduli space, hep-th/9205106 [SPIRES].

[9] M. Kontsevich, Intersection theory on the moduli space of curves and the matrix Airy function, Commun. Math. Phys. 147 (1992) 1 [SPIRES]. 
[10] E. Brézin and S. Hikami, Intersection theory from duality and replica, Commun. Math. Phys. 283 (2008) 507 [arXiv:0708.2210] [SPIRES].

[11] E. Brézin and S. Hikami, Vertices from replica in a random matrix theory, arXiv:0704.2044 [SPIRES].

[12] E. Brézin and S. Hikami, Intersection numbers of Riemann surfaces from Gaussian matrix models, JHEP 10 (2007) 096 [arXiv:0709.3378] [SPIRES].

[13] E. Brézin and S. Hikami, Computing topological invariants with one and two-matrix models, JHEP 04 (2009) 110 [arXiv:0810.1085] [SPIRES].

[14] L. Chekhov and Y. Makeenko, A hint on the external field problem for matrix models, Phys. Lett. B 278 (1992) 271 [hep-th/9202006] [SPIRES].

[15] S. Kharchev, A. Marshakov, A. Mironov and A. Morozov, Generalized Kontsevich model versus Toda hierarchy and discrete matrix models, Nucl. Phys. B 397 (1993) 339 [hep-th/9203043] [SPIRES].

[16] E. Brézin and S. Hikami, Extension of level-spacing universality, Phys. Rev. E 56 (1997) 264 [cond-mat/9702213].

[17] B. de Wit and G. 't Hooft, Nonconvergence of the $1 / N$ expansion for $\mathrm{SU}(N)$ gauge fields on a lattice, Phys. Lett. B 69 (1977) 61 [SPIRES].

[18] E. Witten, Algebraic geometry associated with matrix models of two dimensional gravity, in Topological Methods in Modern Mathematics, Publish or Perish INC, New York U.S.A. (1993).

[19] R.C.Penner, Perturbative series and the moduli space of Riemann surfaces, J. Diff. Geometry 27 (1988) 35.

[20] J.Harer and D.Zagier, The Euler characteristic of the moduli space of curves, Invent. Math. 85 (1986) 457.

[21] E. Witten, String theory and black holes, Phys. Rev.D 44 (1991) 314 [SPIRES].

[22] L. Susskind, Matrix theory black holes and the Gross Witten transition, in Nonperturbative aspects od strings, branes and supersymmetry, Trieste 1998, World Scientific, Singapore (1998) hep-th/9805115 [SPIRES].

[23] L. Álvarez-Gaumé, P. Basu, M. Mariño and S.R. Wadia, Blackhole / string transition for the small Schwarzschild blackhole of $A d S_{5} \times S^{5}$ and critical unitary matrix models, Eur. Phys. J. C 48 (2006) 647 [hep-th/0605041] [SPIRES]. 